Joshua Osowick MBBS Paediatric Infectious Diseases Fellow, ${ }^{1}$ and Honorary Fellow 2

Amanda Gwee MBBS, FRACP, DTMH Paediatric Infectious Diseases Fellow, ' and Honorary Fellow

Jesuina Noronha MBBS, DCH Paediatric Fellow

Pamela Palasanthiran MBBS, MD, FRACP Paediatric Infectious Diseases Physician, ${ }^{4}$ and Conjoint Associate Professor ${ }^{5}$

Brendan McMullan

BMed, FRACP, FRCPA Paediatric Infectious Diseases Physician, ${ }^{4}$ and Conjoint Lecturer $^{5}$

Philip N Britton BMedSci, MBBS, FRACP Paediatric Infectious Diseases Fellow, ${ }^{6}$ and Dean's Fellow

David Isaacs

MD, FRACP FRCPCH Paediatric Infectious Diseases

Physician, ${ }^{6}$ and Clinical Professor $^{7}$

Tony Lai

BPharm, GCClinPharm Antimicrobial Stewardship Pharmacist ${ }^{8}$

Clare Nourse

MRCPI, FRACP, MD Paediatric Infectious Diseases Physician, ${ }^{9}$ and Associate Professor ${ }^{10}$

Minyon Avent BSc(Hons), PharmD, BCPS Antimicrobial Stewardship

Pharmacist," and Clinical

Research Pharmacist

Paul Moriarty

MBBCh, BAO, MD Paediatric Infectious Diseases Fellow ${ }^{9}$

Julia Clark BMBS, FRCPCH, FRACP Paediatric Infectious Disease Physician ${ }^{9}$

Joshua R Francis BAppSc(MedSc), MBBS, FRACP

Paediatric Infectious Diseases Physician13,14

Christopher C Blyth MBBS, FRACP, FRCPA Infectious Diseases Physician and Clinical Microbiologist, ${ }^{13}$ and Associate Professor ${ }^{15}$

Celia M Cooper BMBS, FRACP Infectious Diseases Physician and Clinical Microbiologist ${ }^{16}$

Penelope A Bryant MRCP, FRACP, PhD Paediatric Infectious Diseases

Physician,' and Clinical Associate Professorl?

\title{
Australia-wide point prevalence survey of the use and appropriateness of antimicrobial prescribing for children in hospital
}

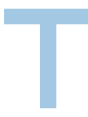
he threat of antimicrobial resistance and its impact on health care settings globally are well recognised, and a multifaceted and coordinated response is required. ${ }^{1-3}$ Antimicrobial use is the main driver of the development of resistance and, as such, advocacy for rational use of existing antimicrobial drugs - antimicrobial stewardship (AMS) - is vital for preventing the development of resistance. 4,5 Very few new antimicrobials are in the drug discovery pipeline so ensuring that the right drug is prescribed in the right dose, via the right route and for the right duration is critical. ${ }^{6}$ In contrast, AMS interventions are readily available and have shown promise in delivering improvement in measures of process and outcome.7,8

Design and implementation of effective and efficient AMS interventions are reliant on data regarding current antimicrobial prescribing patterns. There is a paucity of such data for hospitalised children. ${ }^{9-11} \mathrm{We}$ aimed to address this evidence gap by using a point prevalence survey (PPS) to describe antimicrobial use in hospitalised Australian children, analyse the appropriateness of this antimicrobial use and identify potential opportunities for quality improvement.

\section{Methods}

In conjunction with the Antibiotic Resistance and Prescribing in European Children (ARPEC) study, ${ }^{11}$ eight Australian paediatric hospitals across five states participated in a single-day hospital-wide PPS of antimicrobial prescribing in late spring and early summer 2012: the

\section{Abstract}

Objectives: To describe antimicrobial use in hospitalised Australian children and to analyse the appropriateness of this antimicrobial use.

Design: Multicentre single-day hospital-wide point prevalence survey, conducted in conjunction with the Antimicrobial Resistance and Prescribing in European

Children study.

Setting: Eight children's hospitals across five Australian states, surveyed during late spring and early summer 2012.

Patients: Children and adolescents who were inpatients at 8 am on the day of the survey.

Main outcome measures: Quantity and quality of antimicrobial prescribing.

Results: Of 1373 patients, 631 (46\%) were prescribed at least one antimicrobial agent, 198 (31\%) of whom were $<1$ year old. The highest antimicrobial prescribing rates were in haematology and oncology wards (76\% [95/125]) and paediatric intensive care units (55\% [44/80]). Of 1174 antimicrobial prescriptions, 550 (47\%) were for community-acquired infections, 175 (15\%) were for hospital-acquired infections and 437 (37\%) were for prophylaxis. Empirical treatment accounted for $72 \%$ of antimicrobial prescriptions for community-acquired infections and 58\% for hospital-acquired infections (395 and 102 prescriptions, respectively). A total of 915 prescriptions (78\%) were for antibacterials; antifungals and antivirals were predominantly used for prophylaxis. The most commonly prescribed antibacterials were narrowspectrum penicillins (18\% [164 prescriptions]), $\beta$-lactam- $\beta$-lactamase inhibitor combinations (15\% [136]) and aminoglycosides (14\% [128]). Overall, 957 prescriptions (82\%) were deemed appropriate, but this varied between hospitals (range, 66\% [74/112]) to 95\% [165/174]) and specialties (range, 65\% [122/187] to 94\% [204/217]). Among surgical patients, 65 of 187 antimicrobial prescriptions (35\%) were deemed inappropriate, and a common reason for this was excessive prophylaxis duration.

Conclusion: A point prevalence survey is a useful cross-sectional method for quantifying antimicrobial use in paediatric populations. The value is significantly augmented by adding assessment of prescribing quality.

Royal Children's Hospital Melbourne (Victoria, 14 November 2012), Monash Children's Hospital (Victoria, 22 November 2012), Sydney Children's Hospital (New South Wales, 18 October 2012), Children's Hospital at Westmead (New South Wales, 6 December 2012), Mater Children's Hospital (Queensland, 15 November 2012), Royal Children's Hospital (Queensland, 15 November 2012), Women's and Children's Hospital (South Australia, 14 November 2012) and Princess Margaret Hospital for Children (Western Australia, 16 November 2012). Children and adolescents who were inpatients at $8 \mathrm{am}$ on the day of the survey were included.

De-identified data were collected and entered on standardised data collection forms, which were submitted to the ARPEC web-based data-entry system. Institution and department was recorded for all patients. For those receiving antimicrobials (including antivirals), data on the following were also collected: age; sex; 
1 Infectious Diseases Unit Royal Children's Hospital Melbourne, Melbourne, VIC.

2 Murdoch Childrens Research Institute, Melbourne, VIC

3 Department of Paediatric Infectious Diseases, Monash Children's Hospital, Melbourne, VIC.

4 Department of Immunology and Infectious Diseases, Sydney Children's Hospital, Sydney, NSW.

5 School of Women' and Children's Health, University of New South Wales, Sydney, NSW.

6 Infectious Diseases Department and Microbiology Department Children's Hospital at Westmead, Sydney, NSW.

7 Discipline of Paediatrics and Child Health,

Sydney Medical School, University of Sydney, Sydney, NSW.

8 Pharmacy Department, Children's Hospital at Westmead, Sydney, NSW.

9 Infection Management and Prevention Service, Lad Cilento Children's Hospital, Brisbane, QLD.

10 Discipline of Paediatrics and Child Health, University of Queensland, Brisbane, QLD.

11 Mater Pharmacy, Mater Health Services, Brisbane, QLD.

12 UQ Centre for Clinical Research, University of Queensland, Brisbane, QLD.

13 Department of General Paediatrics, Princess Margaret Hospital for Children, Perth, WA.

14 Department of Paediatrics, Royal Darwin Hospital, Darwin, NT.

15 School of Paediatrics and Child Health, University of Western Australia, Perth WA

16 SA Pathology, Women's and Children's Hospital, Adelaide, SA.

17 Department of Paediatrics, University of Melbourne, Melbourne, VIC.

joshua.osowicki@ rch.org.au

MJA 2014; 201: 657-662 doi: 10.5694/mjal3.00154

Online first 24/11/14 weight; comorbid conditions; antimicrobial drugs that were given (including dose, dosing interval, route of administration and duration of use); whether the indication for antimicrobial treatment was a community- or hospital-acquired infection (with the latter defined as symptoms starting $>48$ hours after admission), or prophylaxis; and whether the antimicrobial treatment was empirical or targeted.

Appropriateness of antimicrobial prescribing was also assessed. At six hospitals, this was determined by two senior infectious diseases physicians and/or AMS pharmacists; at the other two hospitals, one clinician performed this role. Appropriateness was assessed on the basis of the clinical scenario, including microbiological findings, institutional antimicrobial resistance patterns, and institutional treatment guidelines, where available. Standardised terminology was applied to describe appropriateness (Box 1).

Where required, ethics approval was received from respective institutional human research ethics committees.

\section{Results}

\section{Point prevalence survey}

At the eight participating hospitals, the numbers of beds ranged from 120 to 300 and bed occupancy ranged from $62 \%$ to $98 \%$. Of 1373 patients included in the study, 631 (46\%) were prescribed at least one antimicrobial agent (Box 2), or 583 (42\%) if topical agents (eg, orally administered, nonabsorbed drugs such as oral nystatin) are not considered. Of the 631 patients receiving antimicrobials, $143(23 \%)$ were $<3$ months old and 198 (31\%) were $<1$ year old. The most common underlying conditions among those prescribed at least one antimicrobial were haematological and oncological conditions (17\% [106 patients]), noncardiac surgical diseases (13\% [85]) and chronic neurological conditions including cerebral palsy ( $8 \%$ [51]).

The hospital units with the highest rates of antimicrobial prescribing were haematology and oncology wards and paediatric intensive care units (PICUs) (in which, overall, 76\% [95/125] and 55\% [44/80] of patients,
1 Criteria for categorising appropriateness of antimicrobial prescribing* Appropriate decision

- Correct choice of antimicrobial and correct application

- Correct choice of antimicrobial and incorrect application

Inappropriate decision

- No infection, no prophylaxis needed and no antimicrobial needed

No infection, antimicrobial used as prophylaxis and no antimicrobial needed

Inappropriate choice

- Antimicrobial needed but different from the one used - unnecessary diversion from antimicrobial guidelines or considered "too broad"

- Antimicrobial needed but different from the one used - not sufficient for indication or considered "too narrow"

Incorrect application

- Incorrect dose

- Incorrect dosing interval

- Incorrect route of administration

- Incorrect duration of use (too long)

Insufficient information

- No infection, insufficient information on whether antimicrobial was needed

- Insufficient information about infection

Infection present, antimicrobial needed, insufficient information on whether choice and application were correct

* More than one criterion per prescription may apply.

2 Proportions of patients at Australian paediatric hospitals who were and were not prescribed antimicrobials

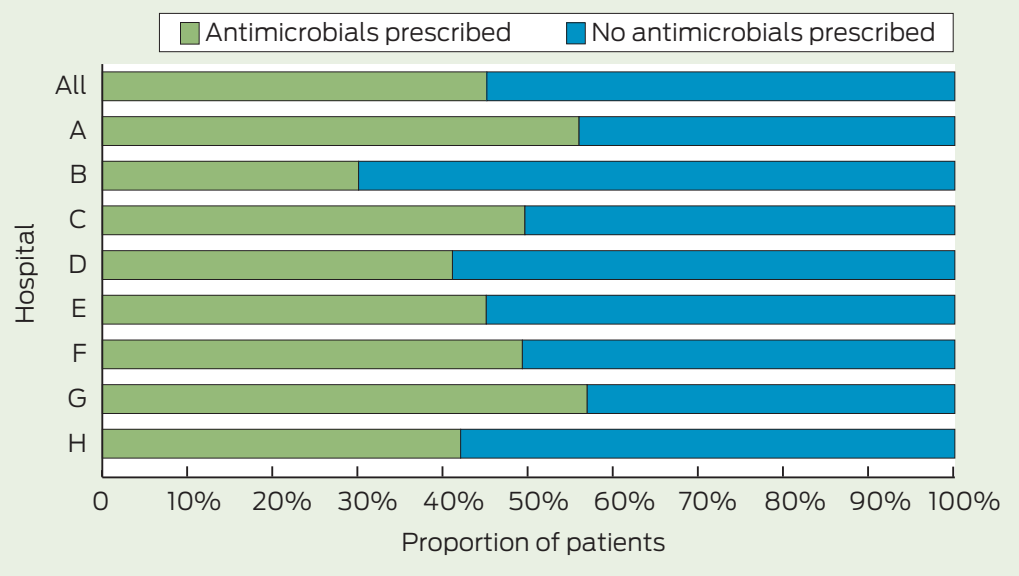

respectively, were receiving $\geqslant 1$ antimicrobial). Neonatal units had the most variation in rates of antimicrobial prescribing (range, 32\% [21/65] to $96 \%$ [23/24]) (Appendix 1; all appendices online at mja.com.au).

There were 1174 antimicrobial prescriptions: 550 (47\%) for community-acquired infections, 175 (15\%) for hospital-acquired infections, 437 (37\%) for prophylaxis (for surgery or a medical condition), and 12 (1\%) for indications that were not recorded. Empirical treatment accounted for $72 \%$ of prescriptions for community-acquired infections (395/550), but only $58 \%$ of prescriptions for hospital-acquired infections $(102 / 175)$.

Of the 550 prescriptions for community-acquired infections, the commonest indications were lower respiratory tract infection $(22 \%$ [122 prescriptions]), surgical infection (13\% [71]) and sepsis (10\% [57]). Of the 175 prescriptions for hospitalacquired infections, the commonest indications were sepsis (18\% [32]), surgical infection (13\% [22]), lower respiratory tract infection $(12 \%$ [21]) and febrile neutropenia (12\% [21]).

The number of antimicrobial prescriptions for all indications accounting for $50 \%$ of use when ranked by 
3 Antimicrobial prescriptions* accounting for $50 \%$ of use when ranked by frequency of prescribing, for patients at Australian paediatric hospitals

\begin{tabular}{|c|c|c|c|c|}
\hline & $\begin{array}{l}\text { No. }(\%) \text { of all antimicrobial } \\
\text { prescriptions }(n=1174)\end{array}$ & No. (\%) used for treatment & $\begin{array}{l}\text { No. used for } \\
\text { targeted treatment }\end{array}$ & $\begin{array}{l}\text { No. (\%) used } \\
\text { for prophylaxis }\end{array}$ \\
\hline Nystatin & $122(10 \%)$ & 0 & Not applicable & $122(100 \%)$ \\
\hline Gentamicin & $102(9 \%)$ & $77(75 \%)$ & 9 & $25(25 \%)$ \\
\hline Trimethoprim-sulfamethoxazole & $87(7 \%)$ & $20(23 \%)$ & 7 & $67(77 \%)$ \\
\hline Amoxycillin or ampicillin & $61(5 \%)$ & $40(66 \%)$ & 3 & $21(34 \%)$ \\
\hline Cefotaxime & $61(5 \%)$ & $51(84 \%)$ & 13 & $10(16 \%)$ \\
\hline Piperacillin-tazobactam & $59(5 \%)$ & $54(92 \%)$ & 9 & $5(8 \%)$ \\
\hline Cephazolin & $57(5 \%)$ & $13(23 \%)$ & 2 & $44(77 \%)$ \\
\hline Flucloxacillin & 51 (4\%) & 48 (94\%) & 21 & $3(6 \%)$ \\
\hline
\end{tabular}

*For all indications.

4 Systemic antibacterial prescriptions for all indications, for patients at Australian paediatric hospitals

\begin{tabular}{|c|c|c|c|c|c|c|c|c|c|}
\hline & \multicolumn{9}{|c|}{ Hospital } \\
\hline & All & A & B & C & $\mathrm{D}$ & $E$ & $\mathrm{~F}$ & G & $\mathrm{H}$ \\
\hline Numbers of patients receiving $\geqslant 1$ systemic antibacterial & 558 & 98 & 65 & 41 & 62 & 47 & 126 & 54 & 65 \\
\hline \multicolumn{10}{|l|}{ Numbers of prescriptions for systemic antibacterials } \\
\hline Total & 912 & 142 & 106 & 72 & 100 & 77 & 222 & 88 & 105 \\
\hline$\beta$-lactam- $\beta$-lactamase inhibitor combinations & 136 & 24 & 13 & 3 & 21 & 13 & 31 & 14 & 17 \\
\hline Aminoglycosides & 128 & 16 & 19 & 12 & 15 & 9 & 29 & 8 & 20 \\
\hline Penicillins (penicillins V and $G$, aminopenicillins) & 113 & 15 & 16 & 18 & 11 & 8 & 22 & 6 & 17 \\
\hline Cephalosporins - 3rd or 4th generation & 93 & 12 & 13 & 11 & 10 & 11 & 13 & 12 & 11 \\
\hline Trimethoprim-sulfamethoxazole & 87 & 21 & 0 & 7 & 4 & 11 & 27 & 9 & 8 \\
\hline Cephalosporins - 1st or 2nd generation & 76 & 20 & 7 & 1 & 9 & 6 & 20 & 8 & 5 \\
\hline Antistaphylococcal penicillins & 51 & 4 & 11 & 1 & 3 & 5 & 15 & 8 & 4 \\
\hline Glycopeptides & 48 & 7 & 5 & 6 & 4 & 1 & 15 & 2 & 8 \\
\hline Macrolides & 41 & 5 & 4 & 3 & 4 & 6 & 11 & 5 & 3 \\
\hline Metronidazole & 37 & 7 & 5 & 4 & 0 & 0 & 15 & 4 & 2 \\
\hline Fluoroquinolones & 29 & 5 & 2 & 0 & 3 & 3 & 11 & 3 & 2 \\
\hline Carbapenems & 18 & 4 & 0 & 4 & 1 & 3 & 1 & 2 & 3 \\
\hline Lincosamides & 18 & 1 & 2 & 1 & 6 & 0 & 4 & 1 & 3 \\
\hline Rifampicin & 8 & 0 & 2 & 0 & 2 & 0 & 4 & 0 & 0 \\
\hline Colistin & 4 & 0 & 1 & 0 & 0 & 0 & 0 & 2 & 1 \\
\hline Tetracyclines & 4 & 0 & 1 & 0 & 0 & 0 & 0 & 3 & 0 \\
\hline Linezolid & 3 & 1 & 0 & 0 & 1 & 0 & 0 & 0 & 1 \\
\hline
\end{tabular}

frequency of prescribing (DU50\% [drug utilisation 50\%]) was eight (Box 3). The number of antimicrobial prescriptions accounting for $90 \%$ of use (DU90\%) was 27.

Of the 1174 prescriptions for antimicrobials, 915 (78\%) were for antibacterials, of which most $(72 \%$ [661 prescriptions]) were for treatment, as opposed to prophylaxis. There were 207 prescriptions for antifungals $(18 \%)$ and 52 prescriptions for antivirals (4\%), both used predominantly for prophylaxis (Appendix 2).

Of the 915 prescriptions for antibacterials, the three most commonly prescribed classes of antibacterials for all indications were: narrow-spectrum penicillins (penicillins $\mathrm{V}$ and $\mathrm{G}$, aminopenicillins and antistaphylococcal penicillins; $18 \%$ [164 prescriptions]), $\beta$-lactam- $\beta$-lactamase inhibitor combinations (15\% [136]) and aminoglycosides (14\% [128]) (Box 4, Appendix 3, Appendix 4). Considering the 661 antibacterial prescriptions that were for treatment, the most commonly prescribed antibacterials were gentamicin (12\% [77]), piperacillin-tazobactam (8\% [54]), cefotaxime (8\% [51]) and flucloxacillin (7\% [48]). Only three of the antibacterial prescriptions were for topical agents.

\section{Appropriateness of prescribing}

Of the 631 patients receiving antimicrobials, 177 (28\%) were receiving at least one prescription that was deemed to be inappropriate. Of the 1174 prescriptions, 957 (82\%) were deemed appropriate. At individual hospitals, the proportion of prescriptions that were appropriate ranged from $66 \%(74 / 112)$ to $95 \%$ (165/174). Similar variation was observed between specialties (range, $65 \%$ [122/187] to $94 \%$ [204/217]) (Box 5). Of the 217 prescriptions deemed inappropriate, for more than one reason in some cases, 65 (30\%) involved an inappropriate decision to 


\section{Appropriateness of antimicrobial prescriptions, by specialty, for patients at} Australian paediatric hospitals

Insufficient information $\square$ Inappropriate choice $\square$ Appropriate decision Inappropriate application $\square$ Inappropriate decision

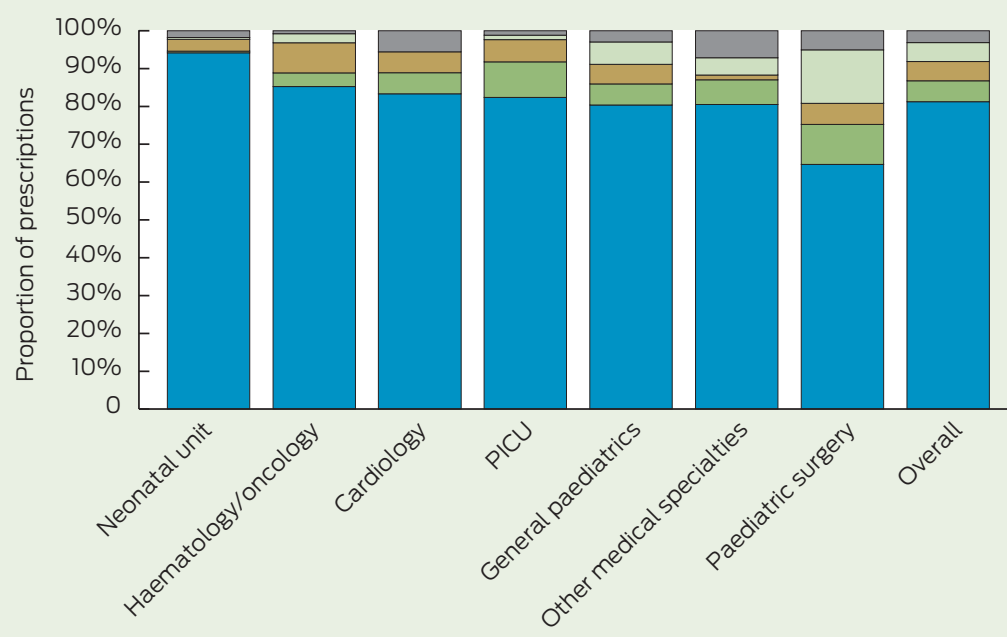

$\mathrm{PICU}=$ paediatric intensive care unit.

use antimicrobials, 60 (28\%) involved an inappropriate choice of antimicrobials, 73 (34\%) involved an inappropriate application, and $32(15 \%)$ lacked sufficient information to assess appropriateness.

The highest rate of prescriptions deemed inappropriate was in surgical patients. Of 131 surgical patients (21\% of patients who received antimicrobials), 53 (40\%) received at least one prescription deemed inappropriate, and this corresponded with 65 of 187 antimicrobial prescriptions for surgical patients (35\%). Of these 65 prescriptions, 21 involved an inappropriate decision, usually to continue perioperative prophylaxis for longer than 24 hours, and 11 involved an inappropriate choice (Box 5).

Antimicrobial prescriptions that were deemed to diverge unnecessarily from antimicrobial guidelines or considered "too broad" included four of 18 prescriptions for carbapenem, seven of 48 for glycopeptides, seven of 93 for third-generation cephalosporins, two of 27 for fluoroquinolones and eight of 136 for $\beta$-lactam- $\beta$ lactamase inhibitor combinations.

\section{Discussion}

This is the first truly representative nationwide PPS in which every standalone children's hospital participated. Very few multicentre surveys have been undertaken in children 9,11,12 and this is the second to incorporate an analysis of appropriateness of antimicrobial prescribing. ${ }^{12}$

The results confirm that antimicrobials are frequently prescribed to children in Australian paediatric hospitals. The overall rate of $46 \%$ is comparable to the average rate of $44 \%$ for hospitalised children in nonEuropean countries and markedly higher than the average rate of $35 \%$ for hospitalised children in European countries. ${ }^{11}$ Our finding that about one-third of patients receiving antimicrobials were $<1$ year old is similar to findings from other studies,, 11 and is related to the higher incidence of bacterial infection in this age group.

Consistent with other surveys of patients, the highest rates of antimicrobial use were in patients with haematological and oncological conditions $(76 \%)$ and patients in PICUs (55\%). Previous surveys in PICUs in the United States, Turkey and Italy have found that $51 \%-76 \%$ of patients were receiving antimicrobials. ${ }^{10,12-14}$ An ARPEC PPS showed similar rates for antibiotic use in PICUs (56\%) and lower rates in haematology and oncology wards (61\%). ${ }^{11}$ AMS intervention studies have focused on these settings and other clinical areas in which antimicrobial use is high.

The most common infections for which antimicrobials were prescribed in our study were similar to those reported in a similar survey in which data on diagnoses were collected. ${ }^{12}$ The relatively low rates of respiratory infection in our study are likely to reflect the time of year in which the data were collected - late spring and early summer, rather than winter. The limited detail beyond site of infection recorded in most surveys of adult inpatients limits attempts at meaningful comparisons. ${ }^{15-17}$

As evidenced by the DU90\%, the range of antimicrobials used was wide. Gentamicin was the most commonly prescribed individual antibacterial agent, reflecting the use of aminoglycosides as first-line therapy in children. This contrasts with the trend in adult medicine, highlighting the importance of paediatric-specific studies. Third- and fourth-generation cephalosporin use, which has been recognised as a driver of resistance, was variable between hospitals, with the differences most likely related to differences in local empirical guidelines (Appendix 3). Low rates of vancomycin, clindamycin and linezolid use reflect the low rates of drug resistance among gram-positive organisms isolated from children at Australian paediatric hospitals. ${ }^{18}$

While a PPS is not adequately powered to determine statistical differences in prescribing patterns between hospitals, the variation between hospitals that we observed likely reflects differences in paediatric tertiary care in Australia, with intensive care, haematology, oncology, and specialty medical and surgical services asymmetrically distributed within cities and between states. ${ }^{4}$ Specialised services such as extracorporeal support, complex surgery for congenital cardiac conditions and haematopoietic stem-cell transplantation are further concentrated in very few hospitals nationwide. Other factors that are likely to have contributed to the variation include the paucity of evidence regarding antimicrobial prescribing for many childhood infections and different local patterns of antimicrobial resistance. The high proportion of empirical prescriptions may reflect difficulty in obtaining high-quality microbiological samples before prescribing antimicrobials in children and the low priority placed on ideal 
specimen collection in some contexts. These factors emphasise the need for specific paediatric guidelines for empirical antimicrobial treatment and for standards to guide timely collection of appropriate microbiological specimens.

In this study, as in everyday practice, appropriateness was determined by local clinicians (infectious diseases physicians and/or pharmacists) familiar with individual patient clinical and microbiological findings, local antimicrobial resistance patterns, institution-specific guidelines for empirical therapy and principles of AMS. Of the eight hospitals that participated, seven had guidelines for empirical treatment of common community-acquired infections of childhood against which appropriateness was judged. ${ }^{4}$ Overall, a high proportion $(82 \%)$ of prescriptions were deemed appropriate. While $28 \%$ of patients received at least one inappropriately prescribed drug, this compared favourably with $47 \%$ of patients in the Turkish survey. ${ }^{12}$

While some of the variation in appropriateness between hospitals in our study $(66 \%-95 \%)$ may represent differences in quality of prescribing, this is unlikely to be a major factor in tertiary children's hospitals throughout the same country. A single-day PPS is not designed to investigate alternative explanations such as differences in AMS resources (eg, different robustness of restricted drug approval systems) and differences in opinions of assessors. Although reporting was standardised in our study, even the clinicians who were experienced in AMS are likely to have differed in how they assessed appropriateness. In a PICU study, the proportion of antimicrobial prescriptions deemed appropriate varied depending on the assessor's specialty: intensivists judged $82 \%$ appropriate; infectious diseases physicians, 69\%; and pharmacists, 45\%. ${ }^{10}$ This may have been because of differences of opinion, or systematic bias in overestimating or underestimating appropriateness depending on differing agendas. In our study, no clear difference was found in the overall assessments between physicians and pharmacists (data not shown).
A PPS is not useful for assessing appropriateness of antimicrobial prescribing against a gold standard (which does not exist for paediatric infections) or for subgroup analyses (for which the format is inadequately powered). Rather, it is useful for identifying under-recognised areas of prescribing that do not meet AMS expectations. Therefore, of more use than differences between hospitals is the analysis between specialties across all hospitals, as this transcends potential individual bias. It is encouraging that haematology and oncology wards and PICUs, which had the highest rates of antimicrobial prescribing, had among the highest rates of appropriateness $(83 \%$ and $82 \%$ of prescriptions, respectively [Box 5]) - a finding mirrored in the Turkish survey.12 However, these specialties frequently have the most patients with complex conditions who are receiving the broadest spectrum antimicrobials, so inappropriate prescribing in these specialties is likely to have the greatest effect on resistance for individual patients, the unit and the hospital. ${ }^{19}$ Therefore ongoing quality improvement endeavours are vital.

Even greater capacity for improvement may lie in areas with lower rates of prescribing but higher patient throughput. With $40 \%$ of surgical patients receiving at least one antimicrobial prescription deemed inappropriate, one area for targeting would be the use of perioperative prophylaxis, for which there are consensus guidelines. ${ }^{20}$ In the Turkish survey, the highest rates of inappropriate prescribing were also in surgical patients, with $80 \%$ of patients receiving at least one inappropriately prescribed drug. ${ }^{12}$ AMS interventions in high-intensity environments often require proportionally intense involvement by AMS practitioners. In less complex areas, such as perioperative prophylaxis, AMS principles may be more easily systematised, with consequent better use of resources. Examples include institutional perioperative prophylaxis protocols with automatic stop orders at 24 hours and a requirement for evidence to support continuation of antimicrobial therapy for longer. The prospect is for AMS principles to be incorporated at a system level in all aspects of antimicrobial prescribing: decision making (to prescribe or not to prescribe antimicrobials), choice of regimen (supported by evidencebased, locally relevant guidelines) and application (particularly duration of use and switching from intravenous to oral administration). ${ }^{21}$

The strengths of this survey lie in its comprehensive nature, with the inclusion of every children's hospital in Australia and every patient receiving antimicrobials. In the absence of electronic prescribing, a PPS is the only way to obtain such a comprehensive picture of antimicrobial use in children. Survey methods that are commonly used for adult patient populations do not take body weight into account, so are inappropriate for use in paediatric patient populations. Survey methods based on numbers of days of therapy are dependent on laborious medical record audits and are therefore usually only viable in settings that use electronic prescribing. A PPS offers relatively highfidelity, cross-sectional quantitative insight into patterns of antimicrobial use. ${ }^{22}$ While labour intensive on the day, it is straightforward and does not require electronic systems, and is therefore appealing in resourcepoor settings. Our survey method was designed by the ARPEC team and piloted across multiple centres for validation purposes. ${ }^{11}$ It provides uniformity of data collection We increased the usefulness of our survey by adding an analysis of appropriateness.

A PPS has several limitations. The cross-sectional nature does not capture duration of antimicrobial therapy or prescribing at hospital discharge. Data that were not collected but would be useful for future surveys include specific infections that were identified and whether suitable microbiological specimens were collected. Also, there is no validated method for assessing appropriateness, and application of the tool by different individuals is likely to result is slight variation. Finally, although patient notes and charts were referred to on the day, subtleties in discussion relating to decision making may have been missed. 
Our study shows the viability and value of multicentre PPSs and appropriateness surveys for acquiring cross-sectional data regarding quantity and quality of antimicrobial prescribing for hospitalised children. It provides a baseline for ongoing audits by AMS teams, conducted in individual hospitals and as multisite collaborations. As hospitals adopt integrated electronic medical record and prescribing systems, there is a new opportunity to incorporate AMS principles into day-to-day hospital work via decision-support algorithms, and subsequently move to a system of continuous prospective monitoring of prescribing patterns. ${ }^{21}$

Acknowledgements: We thank David Andresen, Sean Beggs, Asha Bowen, Nigel Curtis, Andrew Daley, Jacky Dobson, Gabrielle Haeusler, Thomas Snelling, Mike Starr and Lesley Voss - members of the Australasian Stewardship of Antimicrobials in Paediatrics group (part of the Australian and New Zealand Paediatric Infectious Diseases Group) - for their involvement in planning the survey. We also thank the physicians and pharmacists at the participating hospitals, including Maria Chan, Mona Mostaghim and James Newcombe, for assisting with collection of data.

Competing interests: No relevant disclosures.

Received 29 Nov 2013, accepted 23 May 2014.

1 Centers for Disease Control and Prevention. Antibiotic resistance threats in the United States, 2013. Atlanta: CDC, 2013. http://www. cdc.gov/drugresistance/threat-report-2013 (accessed Apr 2014).

2 Ghafur A, Mathai D, Muruganathan A, et al. The Chennai Declaration: a roadmap to tackle the challenge of antimicrobial resistance. Indian J Cancer 2013; 50: 71-73.

3 Laxminarayan R, Duse A, Wattal C, et al. Antibiotic resistance - the need for global solutions. Lancet Infect Dis 2013; 13: 1057-1098.

4 Bryant PA, on behalf of the Australasian Stewardship of Antimicrobials in Paediatrics (ANZPID-ASAP) group. Antimicrobial stewardship resources and activities for children in tertiary hospitals in Australasia: a comprehensive survey. Med J Aust 2015. In press.

5 Newland JG, Hersh AL. Purpose and design of antimicrobial stewardship programs in pediatrics. Pediatr Infect Dis J 2010; 29: 862-863.

6 The Lancet Infectious Diseases. Antibiotic resistance: long-term solutions require action now. Lancet Infect Dis 2013; 13: 995.

7 Davey P, Brown E, Charani E, et al. Interventions to improve antibiotic prescribing practices for hospital inpatients. Cochrane Database Syst Rev 2013; (4): CD003543.

8 Khadem TM, Dodds Ashley E, Wrobel MJ, Brown J. Antimicrobial stewardship: a matter of process or outcome? Pharmacotherapy 2012; 32: 688-706.

9 Amadeo B, Zarb P, Muller A, et al. European Surveillance of Antibiotic Consumption (ESAC) PPS 2008: paediatric antimicrobial prescribing in 32 hospitals of 21 European countries. J Antimicrob Chemother 2010; 65: 2247-2252.

10 Blinova E, Lau E, Bitnun A, et al. Point prevalence survey of antimicrobial utilization in the cardiac and pediatric critical care unit. Pediatr Crit Care Med 2013; 14: e280-e288.

11 Versporten A, Sharland M, Bielicki J, et al. The antibiotic resistance and prescribing in European Children project: a neonatal and pediatric antimicrobial web-based point prevalence survey in 73 hospitals worldwide. Pediatr Infect Dis J 2013; 32: e242-e253.

12 Ceyhan M, Yildirim I, Ecevit C, et al. Inappropriate antimicrobial use in Turkish pediatric hospitals: a multicenter poin prevalence survey. Int J Infect Dis 2009; 14: e55-e6l.
13 Ciofi Degli Atti ML, Raponi M, Tozzi AE, et al. Point prevalence study of antibiotic use in a paediatric hospital in Italy. Euro Surveill 2008; 13: pii: 19003.

14 Grohskopf LA, Huskins WC, Sinkowitz-Cochran RL. Use of antimicrobial agents in United States neonatal and pediatric intensive care patients. Pediatr Infect Dis J 2005: 24: 766-773.

15 Ansari F, Erntell M, Goossens H, Davey P. The European Surveillance of Antimicrobial Consumption (ESAC) point-prevalence survey of antibacterial use in 20 European hospitals in 2006. Clin Infect Dis 2009; 49: 1496-1504.

16 Hosoglu S, Parlak Z, Geyik MF, Palanci Y. Critical evaluation of antimicrobial use - a Turkish university hospital example. J Infect Dev Ctries 2013; 7: 873-879.

17 Zarb P, Goossens H. European Surveillance of Antimicrobial Consumption (ESAC): value of a point-prevalence survey of antimicrobial use across Europe. Drugs 2011; 71: 745-755.

18 Wolf J, Daley AJ, Tilse MH, et al. Antibiotic susceptibility patterns of Staphylococcus aureus isolates from Australian children. J Paediatr Child Health 2010; 46: 404-411.

19 Harbarth S, Samore MH, Lichtenberg D, Carmeli Y. Prolonged antibiotic prophylaxis after cardiovascular surgery and its effect on surgical site infections and antimicrobia resistance. Circulation 2000; 101: 2916-2921.

20 Bratzler DW, Dellinger EP, Olsen KM, et al. Clinical practice guidelines for antimicrobial prophylaxis in surgery. Am J Health Syst Pharm 2013; 70: 195-283.

21 Kullar R, Goff DA, Schulz LT, et al. The "epic" challenge of optimizing antimicrobial stewardship: the role of electronic medical records and technology. Clin Infect Dis 2013; 57: 1005-1013.

22 Porta A, Hsia Y, Doerholt K, et al. Comparing neonatal and paediatric antibiotic prescribing between hospitals: a new algorithm to help international benchmarking. J Antimicrob Chemother 2012; 67: 1278-1286. 